\title{
The Helminth Belt
}

\author{
Carlos Rodriguez ${ }^{1}$ \\ 1 Fundación Jiménez Díaz
}

Potential competing interests: The author(s) declared that no potential competing interests exist.

The helminth belt refers to the ensemble of the world zones where infections with helminth parasites are endemic. The map of the helminth belt usually overlaps with areas where warm and moist climates are predominant and sanitation and hygiene are poor, including temperate zones during warmer months. World regions in the helminth belt comprise most of Africa, Southern Asia (including the Indian Subcontinent) and some patches in South and Central America. 